\title{
AN ELECTRON MICROSCOPICAL EXAMINATION OF THE AGEING PROCESS OF THE WING MUSCLES OF THE HOUSE FLY (MUSCA DOMESTICA)
}

\author{
by \\ P. C. DIEGENBACH \& E. MEIJSSEN-DE RIDDER \\ Zoological Laboratory, University of Amsterdam, The Netherlands
}

\begin{abstract}
Morphometric analysis was carried out on wing muscles of the House Fly (Musca domestica), ranging from the pupation period to death. In the first week a clear increase was found in the percentage of food reserve consumed by the cell components. Both fibrils and sarcosomes increase in circumference during the first week. The sarcosomes then fuse.

After the first week few changes occur. The sarcosomal volume increases in male flies. No disintegration or degeneration of the muscle cells appears with old age. Even the fact that the wing of the male flies exhibits abrasion earlier than that of females cannot be correlated with changes in the wing muscles. The ratio of fibrils to sarcosomes is greater than two to one. This ratio differs in males and females.

A gradual decrease in the number of cristae per sarcosome was observed during ageing. This can influence the total activity of the sarcosome. The succinate dehydrogenase reaction is not dependent on age. The presence of two types of sarcosomes is unlikely. Sarcosomes with more cristae are of a darker colour than those with few cristae.
\end{abstract}

\section{INTRODUCTION}

Morphological changes are often pronounced with increasing age. Disorganization, sickness, old-age pigmentation and other phenomena have been described (Strehler, 1962).

Rockstein \& Bathnagar, 1965, and Rockstein, 1966, studied the size and the number of sarcosomes (mitochondria) in the wing muscles of House Flies (Musca domestica Linnaeus, 1758). They found a maximum in males at approximately 8 days, followed by a noticeable decrease up to 12 days and thereafter a gradual decrease. A maximum was found in females at approximately 8 days, followed by a gradual decrease. The size of the sarcosomes was also maximal (2.28 $\mu \mathrm{m}$ for males, $2.37 \mu \mathrm{m}$ for females) after 8 to 9 days, followed by a slow decrease.
The sarcosomes of Apis mellifica Linnaeus, 1766, increased in 20 days twelve-fold in volume (Herold, 1965). Levenbook \& Williams, 1956, found the number of sarcosomes independent of age in Phormia regina (Meigen, 1826). Dry weight initially increases and then remains constant. The diameter also initially increases (Watanabe \& Williams, 1953), and then remains constant. The resulting diameter of the sarcosomes in female Drosophila melanogaster Meigen, 1830, is larger than those in male specimens.

Degeneration has been described in Lucilia cuprina (Wiedemann, 1830) by Gregory et al., 1968, and in Musca domestica by Simon et al., 1969. The latter distinguished two types of sarcosomes: type $A$ with simple cristae and a light matrix, and type $B$ with many cristae and a dense matrix. They described types A and B as appearing in young flies in which the sarcosomes are loosely spread and in which glycogen is abundant. After three days fenestrae appear in the B sarcosomes, becoming more distinct up to the 15th day. After nine days the number of $B$ sarcosomes clearly increases, and after twelve days the B type dominates. According to Simon et al., 1969, degeneration begins after twenty days. Type A degenerates, with swellings in the intracristate spaces, whereas structureless areas appear in type B. The outer membrane is lost and large open spots appear in the muscle cells. According to Simon et al., 1969, hardly any A sarcosomes are visible after 27 days.

We have examined the structural changes which occur during the ageing of the wing muscles of Musca domestica. Male flies exhibit earlier outward signs of ageing, such as wing abrasion and die earlier than females. We have examined the possibility of correlating wing 
abrasion with a deterioration of muscle components, such as muscle fibrils, sarcosomes, food reserves, or with a deterioration of the muscle itself. Michejda, 1964, noticed that the ratio in percentage of fibrils to percentage of sarcosomes was approximately 1 in animals showing good flying capacity and approximately 2 in animals with poor flying capacity. We examined this ratio in male and female flies to see if any of the components increased or decreased during ageing.

The activity of various enzymes during the ageing process was studied. There is usually a quick increase in activity followed by a gradual decrease. We examined the following possibilities: (1) of a correlation between the changes in activity and the number of cristae, and (2) of changes in the activity of the cristae through the localization of a single enzyme, succinate dehydrogenase. Survival graphs were drawn as well.

\section{METHODS}

\section{A. The breeding of flies}

Approximately 500 flies (Musca domestica) were kept in a cage measuring $40 \times 40 \times 190 \mathrm{~cm}$ which was illuminated for 12 hours per 24 hour period and kept at a temperature of $25^{\circ} \mathrm{C}$. The relative humidity was kept between 50 and 60 percent. Each population was of equal age, having emerged within a 4 hours period. The animals were fed with a mixture of $100 \mathrm{~g}$ sucrose, $100 \mathrm{~g}$ skim-milk powder and $20 \mathrm{~g}$ dried yeast. A supply of drinking water was provided in a petri dish in which a piece of perforated filter paper floated. This filter paper was soaked in paraffin wax (melting point $55^{\circ} \mathrm{C}$ ) to prevent the flies from drowning.

Eggs were collected by placing a mixture of 25 g skim-milk powder, $20 \mathrm{~g}$ cotton wool and $100 \mathrm{ml}$ water in a petri dish, thus giving the flies the opportunity to creep under the wool and deposit their eggs. Clusters of eggs were then placed on a larval medium and covered with about $5 \mathrm{~cm}$ sawdust. Kept at a temperature of $25^{\circ} \mathrm{C}$, the eggs hatched after approximately 14 days. The larval medium consisted of $20 \mathrm{~g}$ agar in 11 water, $100 \mathrm{~g}$ whole milk powder and $100 \mathrm{~g}$ dried yeast. The dried yeast and milk were added once the agar/water solution had sufficiently cooled, after which the mixture was cooked again. In order to prevent moulding, $10 \mathrm{ml} \mathrm{10 \%} \mathrm{nipagine} \mathrm{in} \mathrm{ethanol}$ was added to every $1000 \mathrm{ml}$ of the medium. A layer of approximately $5 \mathrm{~cm}$ of this fluid medium was then poured into 11 herring jars.

\section{B. Survival graphs}

Three times a week the dead flies were removed, counted and their sex was determined. The survival percentage was plotted against time.

\section{Random samples}

Two males and two females of equal age were used from each breeding. In most instances five electron microscope photographs were made and morphometrically analyzed per specimen. The number of specimens and photographs is indicated in table $\mathrm{I}$. The random samples were not alike

Table I. Number of random samples taken at different ages. Indicated are the number of male and female flies sampled at random from the cages and the number of micrographs that were morphometrically evaluated. The random samples are not alike in every age group due to mishaps during the procedure and to a shortage of old flies.

\begin{tabular}{lcccc}
\hline & \multicolumn{2}{c}{$\begin{array}{c}\delta \\
\text { number of } \\
\text { age }\end{array}$} & flies & \multicolumn{2}{c}{$\begin{array}{c}\text { } \\
\text { photographs }\end{array}$} & flies & photographs \\
\hline 0-5 min. & 10 & 50 & 9 & 44 \\
1 day & 13 & 65 & 6 & 30 \\
6 days & 13 & 65 & 8 & 39 \\
15 days & 13 & 65 & 7 & 35 \\
22 days & 13 & 64 & 7 & 34 \\
28 days & 13 & 65 & 6 & 30 \\
36 days & 8 & 41 & 7 & 35 \\
42 days & 5 & 25 & 7 & 35 \\
49 days & 3 & 15 & 5 & 25 \\
54 days & - & - & 3 & 15 \\
\hline
\end{tabular}

in every case due to mishaps during the procedure and to a shortage of old flies. Fewer females than males were used. The random samples were taken as follows: two male flies, one with and one without wings, and two females were arbitrarily chosen. It was previously decided upon which flies would be used. They were then relatively easily captured. This excluded the possibility that only inert and sick animals would be used in the random samples.

\section{The preparation and embedding of the wing muscles}

The flies were immobilized by cooling in a 
refrigerator, without using $\mathrm{CO}_{2}$. Each animal was decapitated and the dorsal thorax was cut open. The six fibres of the musculus dorsalis, responsible for the downbeat of the wings, are then clearly seen. The thorax was fastened with pins, sprinkled with a fixing fluid and kept at a temperature of $4{ }^{\circ} \mathrm{C}$ for 1 hour. A solution of $2.5 \%$ glutaraldehyde in $0.05 \mathrm{M}$ cacodylate buffer (pH 7.4) with $0.17 \mathrm{M}$ sucrose added, was used as a fixing fluid. The most ventral muscle was cut out and rinsed overnight in $0.1 \mathrm{M}$ cacodylate ( $\mathrm{pH}$ 7.4) with $0.34 \mathrm{M}$ sucrose.

The following day postfixation was carried out in $1 \%$ osmium tetroxyde for one hour, followed by half an hour staining with $1 \%$ uranyl nitrate. After rinsing with distilled water, the muscle was dehydrated and embedded in Epon. Ultrathin sections were stained with lead tartrate. Sections to be used for enzymatic reaction tests were treated for 1 minute with alcoholic uranyl acetate in order to prevent the basic lead staining from affecting the precipitation. The uranyl treatment was followed by the lead staining. The incubation time for the succinate dehydrogenase reaction was 10-15 minutes; then the sections were rinsed for a short time with a phosphate buffer and fixed as described above. The muscles were incubated in either the Ogawa et al., 1968, medium or in the medium according to KerpelFronius \& Hajos, 1968.

Control reactions were carried out by replacing the substrate solution with a buffer solution. Either $10 \mathrm{mM}$ sodium malonate was used during the incubation as an inhibitor, or, to cope with the slow diffusion, 0.1 $M$ sodium malonate was used 30 minutes previous to and during the incubation.

\section{E. Morphometry}

All measurements were made on muscle cross sections. The criterion for a cross section is the visibility of a hole in the myosin filament. When the section thickness is approximately $60 \mathrm{~nm}$ and the diameter of a myosin filament $10-16 \mathrm{~nm}$, excluding the wall thickness, the maximum deviation of the desired cutting angle was $10-15^{\circ}$. When we estimate the wall thickness at $1 / 4$ of the total cross section, then we find $5-8^{\circ}$ as the maximum deviation from the desired cross section. The maximum error in calculating the surface is then $1 \%$. Five electron micrographs were made per tissue cube at a magnification of
$40,000 \times$, with a Zeiss EM 9 electron microscope. Kodak bromide paper, which is reasonably stable in dimensions, was used as photographic enlarging paper. Fibrils, sarcosomes and the glycogen-like material were cut out of the micrographs. The rest of the sarcoplasm consists of matrix with some diads and small tracheoles. The percentage of the components was estimated by weighing the pieces of paper.

To estimate the number of myosine filaments in a fibril, a piece of the photograph representing $10 \times 10$ filaments is cut for a total of 100 filaments per piece. The number of filaments per fibril is the mean area of the fibrils, divided by the mean area of the filaments, times the number of filaments cut out. In a similar way, the number of cristae per sarcosome and sarcosome length were estimated from longitudinal sections. The number of cristae was estimated by counting over $10 \mathrm{~mm}$ along a line perpendicular to the cristae. The greatest sarcosome length was estimated parallel to the filaments.

\section{RESULTS}

\section{A. General}

If the eggs are produced in the same space of time, that is, within a 4 hours interval, the flies will emerge from the pupa within a period of 4 days. During the first day, flies that emerge are almost exclusively males. The second and third day the majority of the flies, both male and female, pupate and the fourth day a small number of females pupate. Only animals from the second and third day of the pupating period were placed in a cage containing an equal number of male and female flies. When the fly has just hatched, the wings are folded and the wing muscles are white, becoming red with increasing age. The flies do not eat, drink, or fly during the first 24 hours. Clark \& Rockstein, 1964, have named this period, during which many changes occur in the body, "metachemogenesis".

During the rest of the first week the flies eat considerable quantities; later the food intake decreases. Some of the male wings already show abrasion on the third day after pupation. However, wing abrasion was not as apparent as observed by Rockstein \& Bhatnagar, 1965, and Rockstein, 1966. We found quite old males, i.e. 
32 days old, with almost completely intact wings. The wings of females usually remained intact with perhaps some ravelling. As far as we could determine, flies which had lost their wings retained the capacity to contract their muscles. They could still flap their wing remnants.

\section{B. Survival graphs}

The flies live approximately 58 days at a temperature of about $20^{\circ} \mathrm{C}$. The maximum life expectancy was approximately 47 days at a constant temperature of $25^{\circ} \mathrm{C}$ and a high population density (viz. $3250 \mathrm{flies} / \mathrm{m}^{3}$ ). The majority of the flies die in the third week. This corresponds with the results obtained by Curtis, 1963, after the application of a chemical stress. It is not clear whether food competion is the cause of such a short life duration, or whether infection also plays a part. Many loose legs, heads and wings were found in the cages.

A lower population density has a favorable

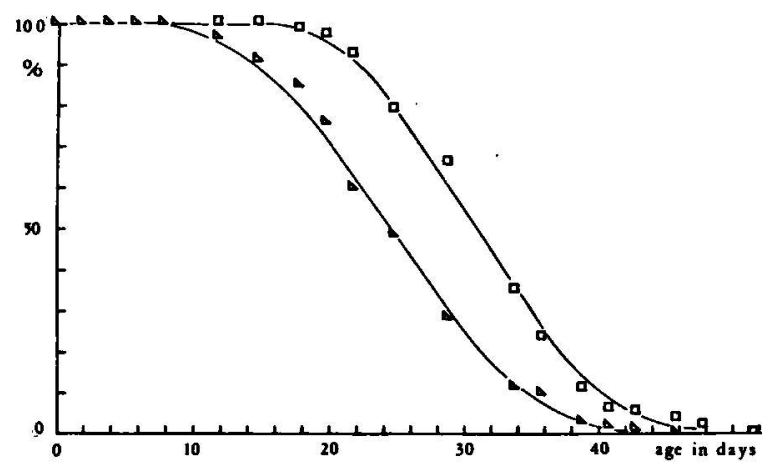

Fig. 1. Percentage survival graph of Musca domestica at a constant temperature of $25^{\circ} \mathrm{C}$ and a population density of 500. Male flies indicated by triangles, females by squares.

effect on the life expectancy. The steep part of the graph moves to the right with decreasing density. Female flies usually live longer than males (fig. 1).

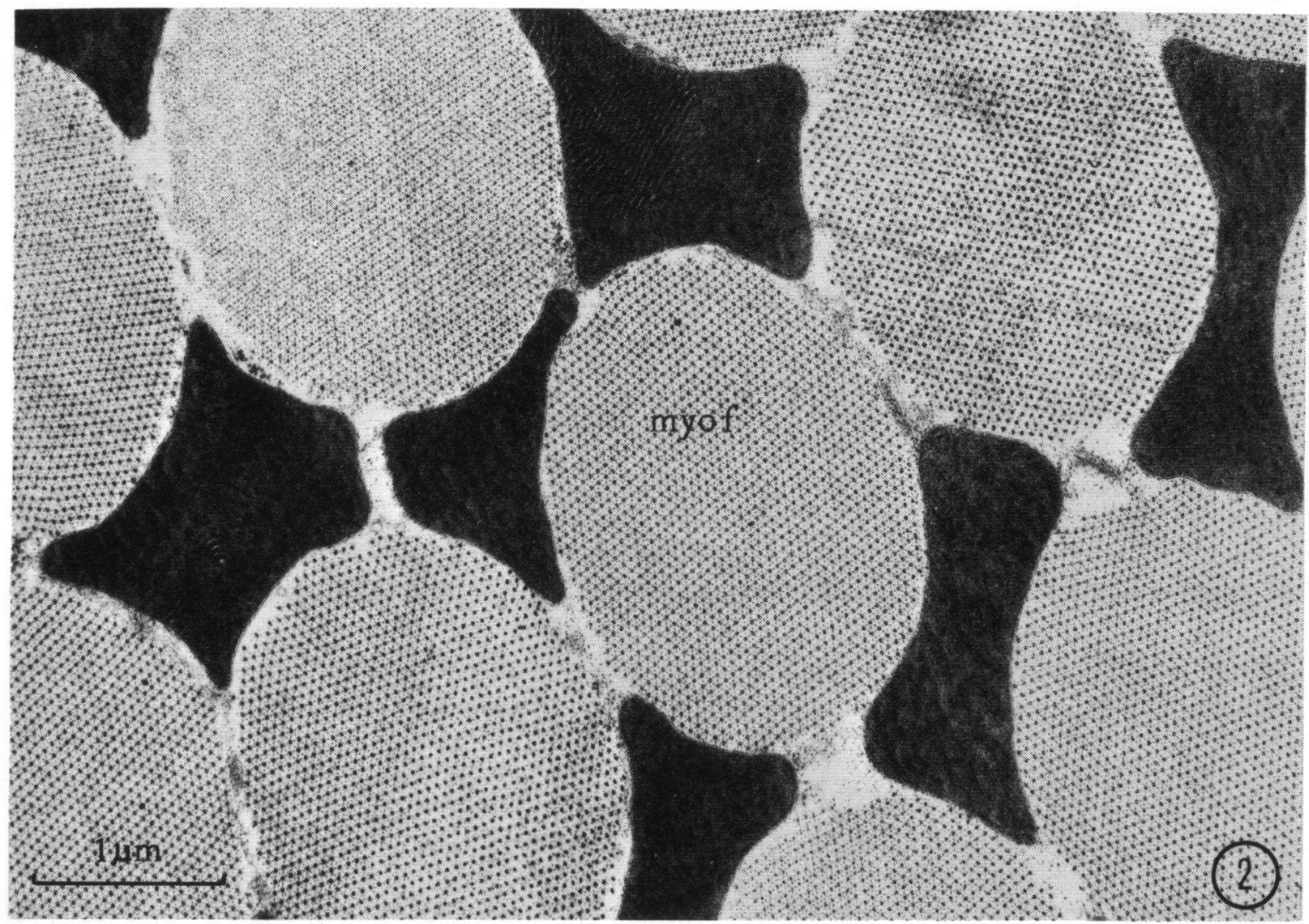

Fig. 2. Transverse section through flight muscle of just pupated flies (Musca domestica), $23500 \times$. In the cristae, the fenestrae are visible. Glycogen is abundant. myof $=$ myofibrils. 


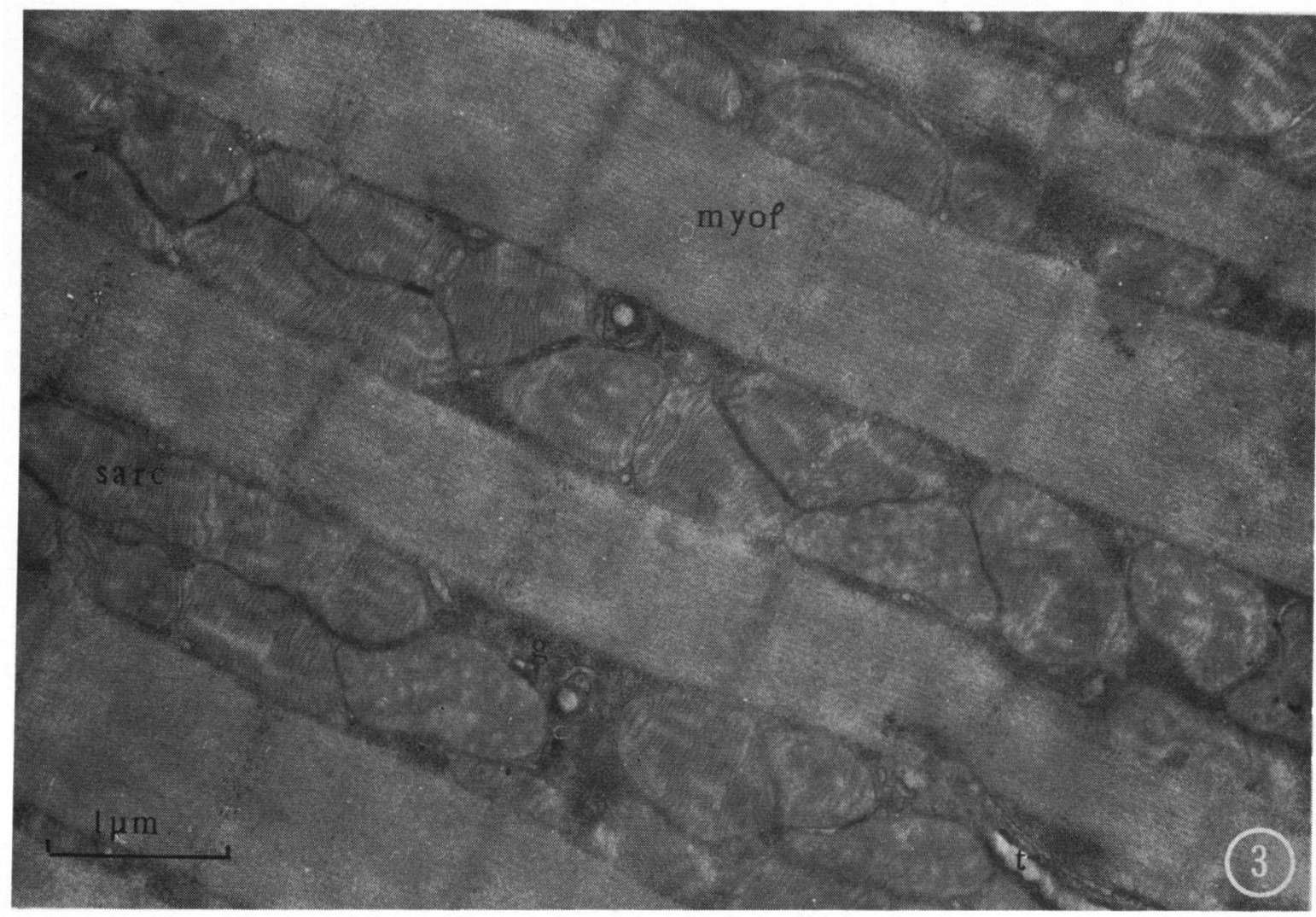

Fig. 3. Longitudinal section through flight muscle of Musca domestica (one day old), $22000 \times$. In this stage the amount of glycogen is generally much higher.

$\mathrm{g}=$ glycogen $;$ myof $=$ myofibrils; sarc $=$ sarcosomes $; \mathrm{t}=$ tracheole.

\section{Electron microscopy of cross sections of wing muscles}

I. 0-5 minutes (fig. 2).

All the muscle components, the fibrils, sarcosomes, food reserves and the remaining sarcoplasma, lie close together. The food reserve is tightly packed, many tracheoles and diads are visible and many small sarcosomes are present. The sarcosomes have few cristae but the fenestrae are already visible, contrary to the observations of Simon et al., 1969. The fibrils are small and the actin and myosin filaments lie notably closer together than in older flies. The cells are small and many nuclei are discernible.

\section{1 day (fig. 3).}

After the first day the muscle components are clearly less crowded together. The food reserve granules also lie farther apart. However, many diads, tracheoles and nuclei are visible. The sarcosomes are small but more cristae and more fenestrae are visible. The distance between the actin and the myosin filaments has increased, while the fibrils are still small.

\section{6 days.}

The amount of food reserve has decreased rapidly. The tracheoles decrease in number per volume unit, while many diads remain. The sarcosomes are much larger and filled with cristae with many fenestrae. The cells, as seen in micrographs of cross sections, are much larger and few nuclei are to be found.

IV. 15 days and older (figs. $4 \& 5 ; 36$ days).

Occasionally sarcosomes with very few cristae are found in the wing muscles of flies 15 days and older. Due to the absence of cristae these sarcosomes are lighter than others. No food reserves are apparent in the majority of the micrographs and no other visible changes occur. Visible signs of degeneration were found in only a few cases of old age. 


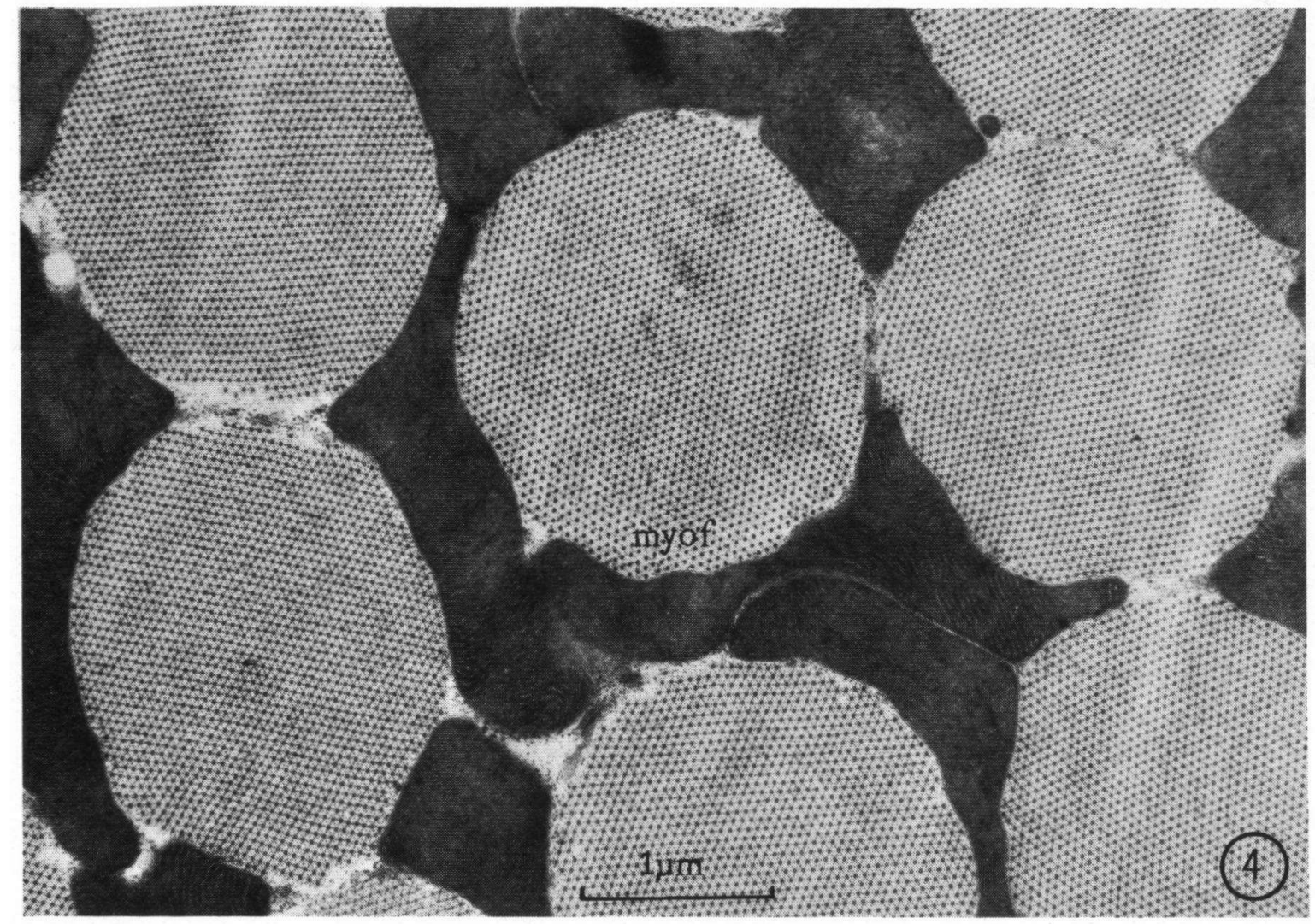

Fig. 4. Cross section through flight muscle of Musca domestica (age 36 days), $23000 \times$. myof $=$ myofibrils.

\section{The muscle components (fig. 6)}

The area occupied by the fibrils increases during the first 6 days. After 6 days it remains practically constant at $62-64 \%$ of the total surface. The sarcosomes occupy about $25 \%$ of the surface (somewhat more in females than in males). The glycogen-like food reserve initially occupies approximately $13 \%$.

In females after 6 days, little is left of the food reserve $(0.2 \%)$, which remains at a low level. In males, the approximately $3 \%$ which remains after six days, completely disappears after 28 days. The space available, due to the disappearance of the food reserve, is used to a large extent for the growth of the fibrils and, to a lesser extent, for the growth of the sarcosomes.

The values we have found for animals younger than 5 minutes indicate that the muscles are more or less pressed together whereby the distance between the actin and the myosin is very small, the food reserve is closely packed together and the sarcosomes occupy a relatively large part of the fiber.

\section{E. The ratio of the fibril percentage to the sarco- some percentage (fig. 7)}

The ratio of fibrils to sarcosomes in male flies is between 2.2 and 2.6. No significant increase occurs during growing (rank correlation test of Kendall) (fig. 7). The ratio declines in females from 2.2 ( 1 day old) to 2.0. This decline is significant (at least when 5 minutes old flies are not included; the last observation was omitted due to the small number of samples).

\section{F. Sarcosomes (figs. 8 \& 9)}

The area of the cross section of sarcosomes increases rapidly during the first 6 days, that is, from $8760 \mu \mathrm{m}^{2}$ to $33200 \mu \mathrm{m}^{2}$ in males and from $11040 \mu \mathrm{m}^{2}$ to $33240 \mu \mathrm{m}^{2}$ in females. The sarcosomes continue to grow (this growth is significant 


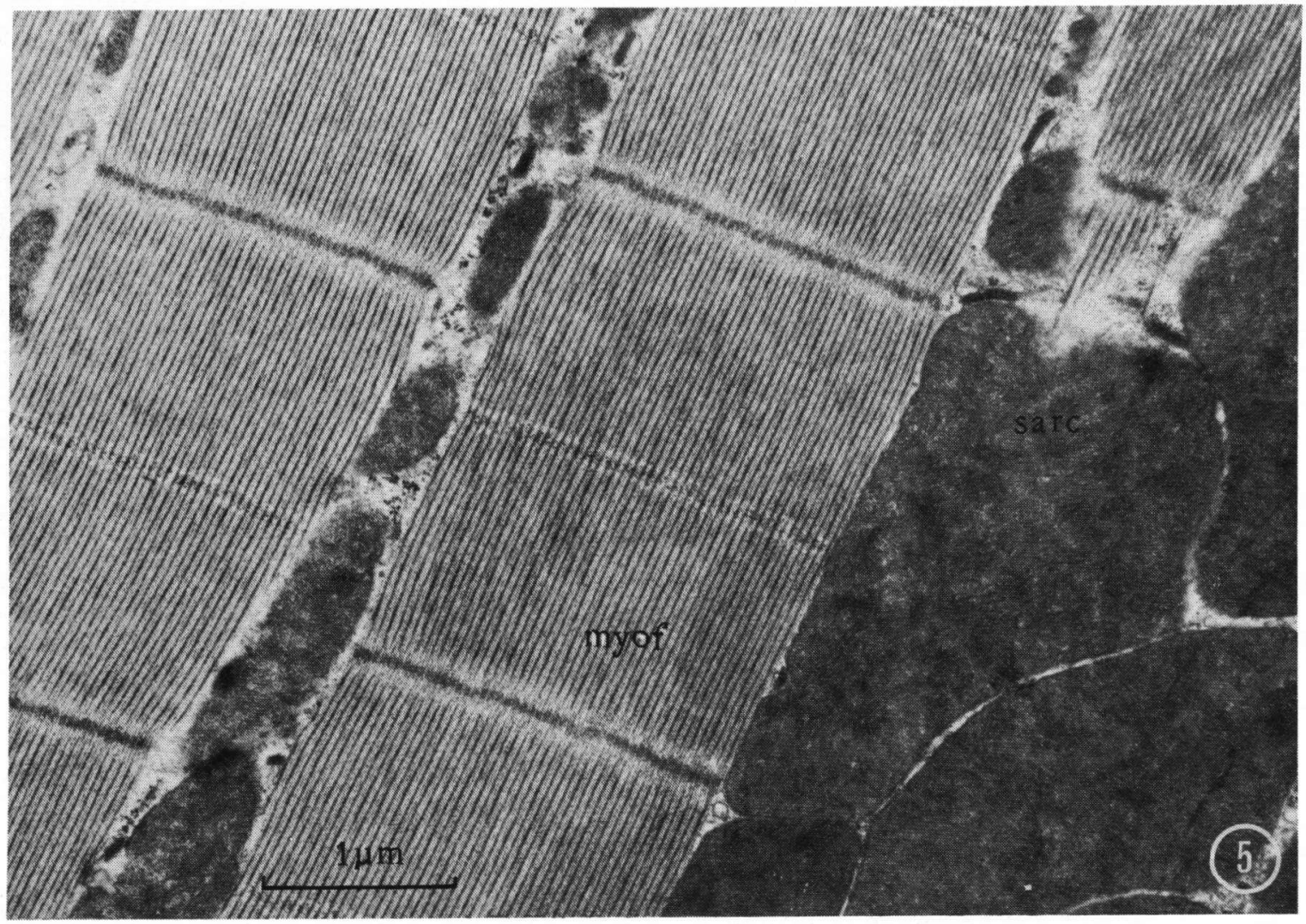

Fig. 5. Longitudinal section through flight muscle of Musca domestica (age 36 days), $23000 \times$. myof $=$ myofibrils; sarc $=$ sarcosomes.

in males) (fig. 8). This in contrary to Rockstein's, 1966 , observations.

After approximately 28 days an increase to a maximum of $47170 \mu \mathrm{m}^{2}$ is apparent in males. Although the percentage of the area occupied by sarcosomes hardly changes (fig. 6), their number declines sharply, while sarcosomes regularly appear with a membrane through the sarcosome. The sarcosomes are now perhaps larger due to the joining of two sarcosomes.

The length of the sarcosomes was measured in longitudinal sections. It thus appears (fig. 9) that the sarcosomes increase in length during the first 6 days and shorten during the following 2 weeks. After 28 days large sarcosomes appear once again. This phenomenon is similar to that observed in cross sections. Thus, the sarcosomes increase in volume during the first week, followed by a decrease during the second and third weeks. This corresponds with Rockstein's, 1966, findings. Thereafter a strong increase occurred while Rockstein observed a decrease.
However, in determining the activity of sarcosomes, not only the volume is of importance, but also the number of cristae. Thus the distance between the cristae was determined for the same sarcosomes (male flies only). The density decreases up to 28 days from $0.053 \mu \mathrm{m}$ between the cristae to $0.035 \mu \mathrm{m}$. After 49 days, sarcosomes had a distance between the cristae of $0.039 \mu \mathrm{m}$.

\section{G. The filaments (fig. 10)}

The majority of theories dealing with muscle contraction (Davies, 1963; Ebashi \& Endo, 1968), proceed from the point that the distance between myosin and actin filaments is constant. The results of area measurements for 100 filaments in cross section are shown in fig. 10. Five minutes after emerging the fibrils appear to be pressed together. Thereafter, the data for male and female flies deviate considerably. The increase in distance between myosin filaments in old males, and the decrease between the 15 th and 22 nd day in females, is striking. Both sexes were fixed and 


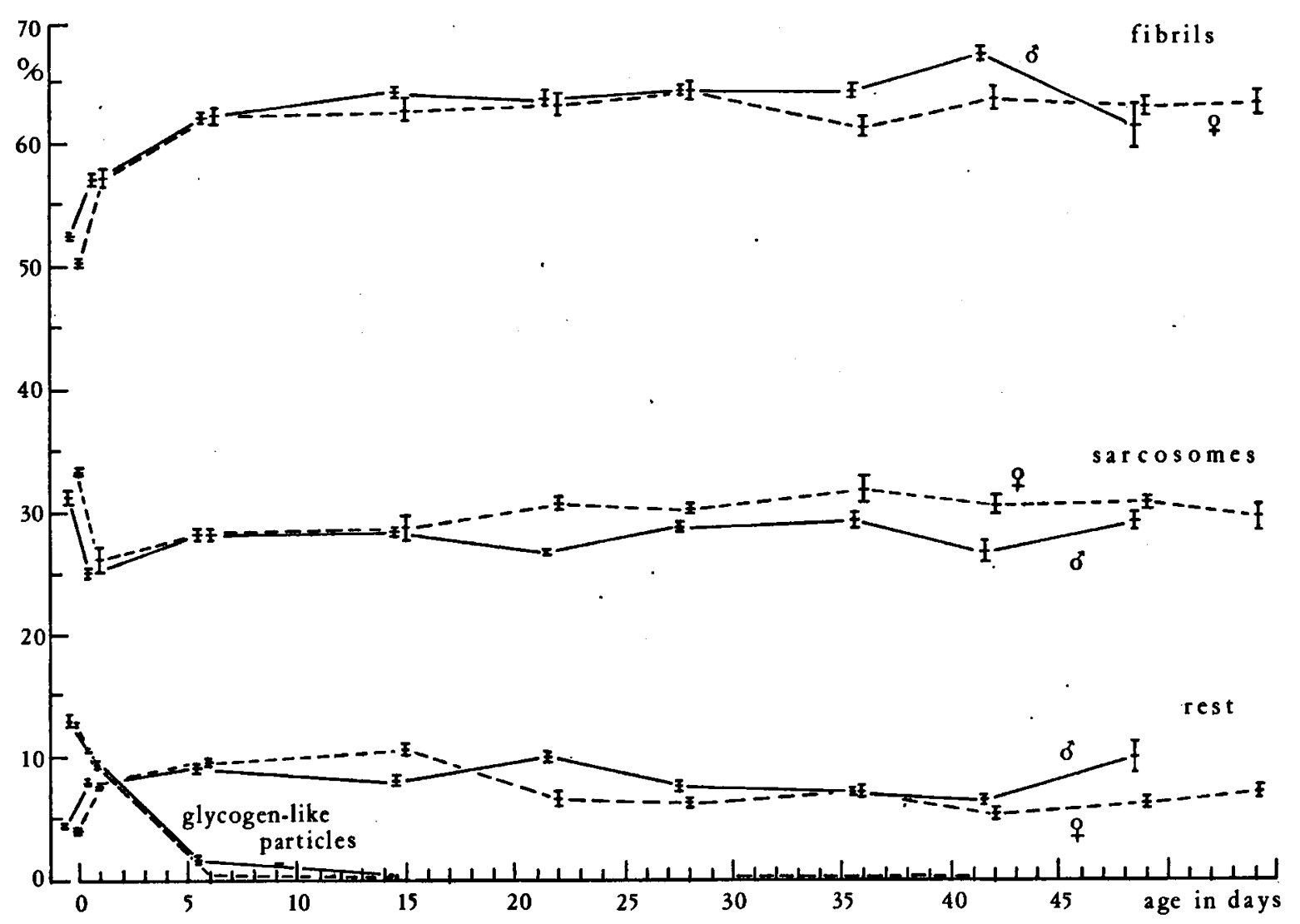

Fig. 6. Distribution of fibrils, sarcosomes, glycogen and glycogen-like particles and other materials in percentage of the total, for flight muscles of $\mathbf{M u s c a}$ domestica at various ages. In this and the following graphs the mean and the $95 \%$ confidence limits of the mean are indicated. Dashed lines indicate the values obtained from females, unbroken lines from male flies.

treated at the same time, thus practically eliminating a difference due to treatment. However, a difference in the distance between the filaments after fixation can result from a difference in osmotic values of the tissues of each sex. Such a difference is possible in relation to the presence of a female protein. The greatest distance was found in old male and young female flies. As these animals are also the easiest to capture, perhaps there exists a connection between the flying capacity or flying behavior and the distance between the filaments.

Newly emerged flies have characteristics different from older ones. The area occupied by fibrils in cross section increases with an increase in the filament distance. The other cell components do not increase (fig. 6). This indicates that the change in filament distance observed should not be due to an artefact. It is clear that the difference in distance between the filaments influences the size of the fibrils. Therefore, we have compared the number of myosin filaments per fibril in animals of various ages. In the first week there is an increase in the number of filaments, thereafter the number remains approximately constant.

\section{H. Enzyme reactions}

Methods recommended by Ogawa et al., 1968, and Kerpel-Fronius \& Hajos, 1968, were used to localize succinate dehydrogenase. The latter method gives the clearest results. Van den Bergh, 1964, has observed that the sarcosomes of a fly have a strong permeability barrier against exogenous substrata. Since it is possible that phosphate or citrate plays an important role in this barrier, the phosphate buffer was replaced by a tris buffer and the citrate either omitted (Ogawa et al., 1968) or replaced by sodium-potassium tartrate.

No reaction products were seen with the Ogawa et al., 1968, method. However, following 


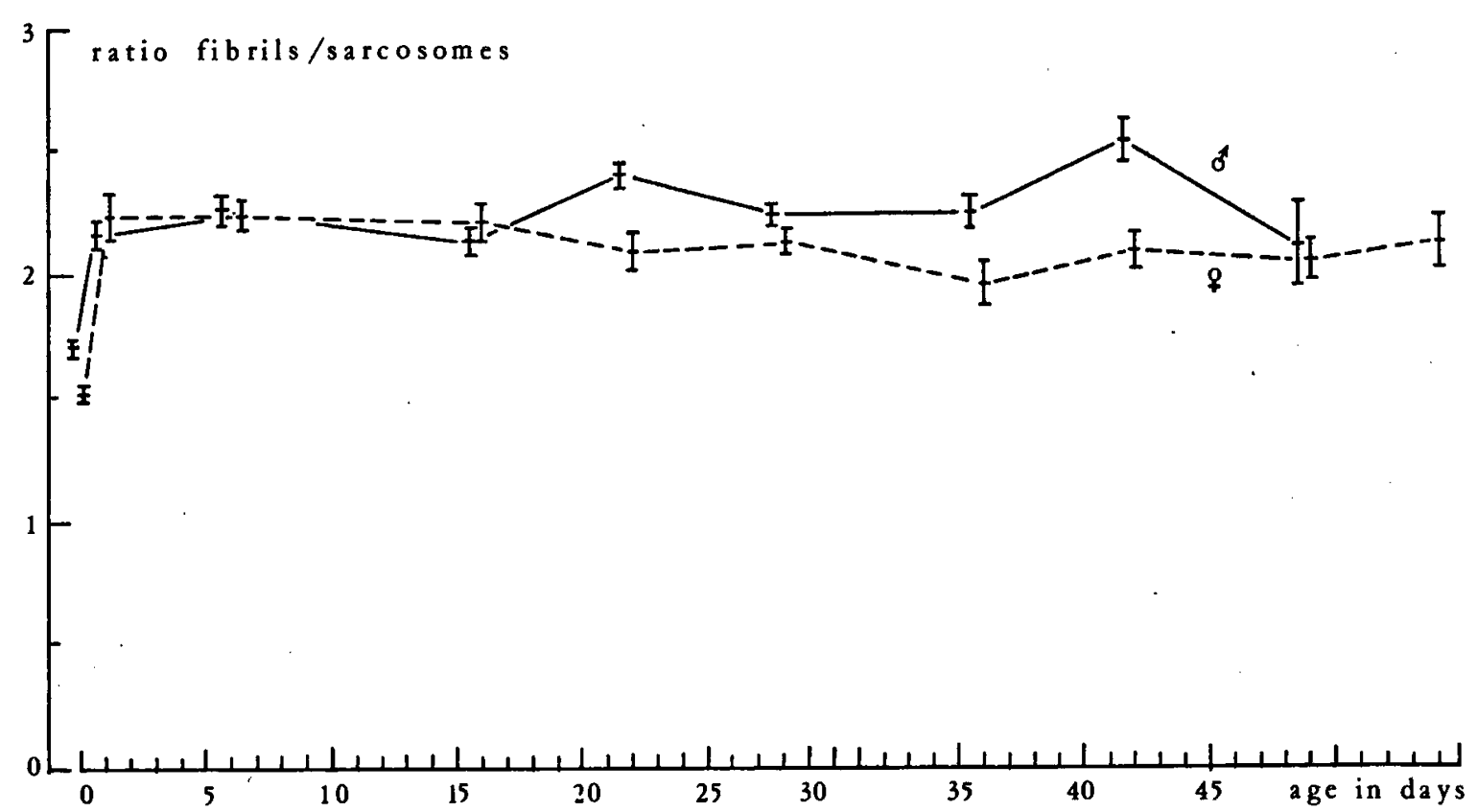

Fig. 7. Ratio fibrils to sarcosomes, for flight muscles of Musca domestica at various ages. Bars represent the $95 \%$ confidence limits of the mean.

the Kerpel-Fronius \& Hajos, 1968, method, we were able to find (the cell membrane being present) a scattered reaction within sarcosomes. Whenever cell membranes were damaged, areas were found within the cell where the outer walls of the sarcosomes were heavily stained and the intracristate spaces, as well as the space between the inner and outer sarcosomal membrane, were completely filled with precipitates. The $Z$ band also showed a reaction. As a positive control, the muscle tissue of a rat heart was used, from which a strong reaction always occurs in which the total intracristate space is filled. However, not all sarcosomes in the same section react as strongly.

Sodium malonate was almost always a complete inhibitor. Omission of the substrate gave (perhaps due to endogenous substrate), fluctuating results. The adding of calcium ions, in order to increase the permeability, was not effective. Previous fixation with hydroxyadipaldehyde inhibited so strongly that the reaction was negative. There was an indication that an increase of the substrate concentration would give better results. When, according to the Ogawa et al. method, the succinate was replaced by glycerophosphate, the only darkening was in the outer membrane, in the fibrils and in the $Z$ band.

The sarcoplasma, which should contain the glycerophosphate dehydrogenase, never showed a reaction. However, it is known that heavy metals strongly inhibit this enzyme. Perhaps the ferricyanide that was used is responsible for this inhibition.

A few tentative conclusions can be made from the enzyme studies. The reaction speed does not appear to be dependent upon age. Only sarcosomes of just emerged and those of one day old flies exhibited little reaction. Heterogeneity in sarcosomes of flies does not appear probable, in contrast with the heart muscle of vertebrates. Only in two 35 days old males heterogenous sarcosomes were found.

\section{DISCUSSION}

\section{A. Representative random samples}

One of the greatest difficulties inherent in a study as described above, is the choosing of an a-select random sample. The flies captured must be representative of the entire population. Various possibilities for choosing flies in an a-select way were considered: such as anaesthesia with $\mathrm{CO}_{2}$ or the placing of the entire cage at $4^{\circ} \mathrm{C}$. However, these methods are quite drastic when they must be repeated every week, during which the death 


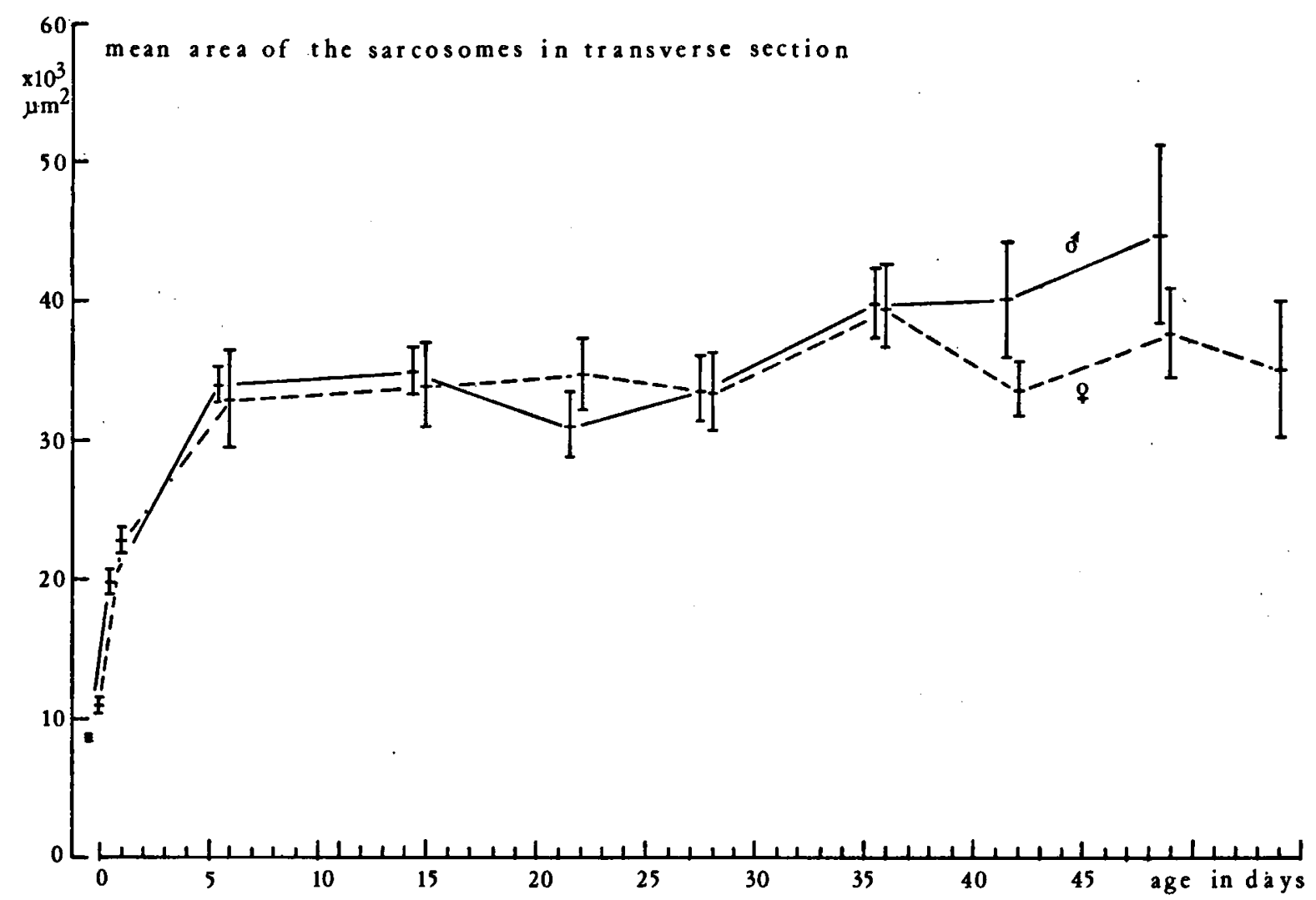

Fig. 8. Mean area of the sarcosomes in transverse sections of flight muscles of Musca domestica at various ages. Bars represent the $95 \%$ confidence limits of the mean.

rate will be much higher than normal. Moreover, older flies are anaesthetized more often than younger ones, a factor which perhaps influences the ageing process. Another method is to use every 10 th or 50 th fly which passes through the cage door. This method is also imperfect especially from a technical point of view. Solitary breeding of flies presents insurmountable technical difficulties. Our method was chosen as a compromise.

\section{B. Structural changes in the wing muscles during the life span}

A distinct growth in muscle components occurs during the first week. Only the amount of food reserve decreases (fig. 6). This has been observed in various insects by several investigators (among others: Herold, 1965, and Rockstein, 1966). This condition remained more or less constant after the first week.

There was no clear degeneration present even in older flies. The disorganization of the fibrils, which Gregory et al., 1968, observed in Lucilia, is out of the question. The irregular contour of older fibrils can be better explained by an uneven increase of filaments than by a disintegration of the outer side, as Gregory et al., 1968, postulate. The fibril of a 6 days old fly is not distinguishable from that of a 49 days old fly. Neither do the sarcosomes degenerate, nor do they loose their outer membrane, as Simon et al., 1969, observed in Musca domestica, nor do they deteriorate. They do not swell either as Gregory et al., 1968, observed in Lucilia.

At most, the number of cristae decreases in some of the sarcosomes. In contradiction to our findings, Sohal \& Allison, 1971, also come to the conclusion that there is a degeneration: the striation pattern, as well as myofibrillar arrangement is lost; sarcosomes in some regions are extremely swollen. Their flies had a much shorter life span than ours. In our opinion, the results arrived at by Gregory et al., 1968, Simon et al., 


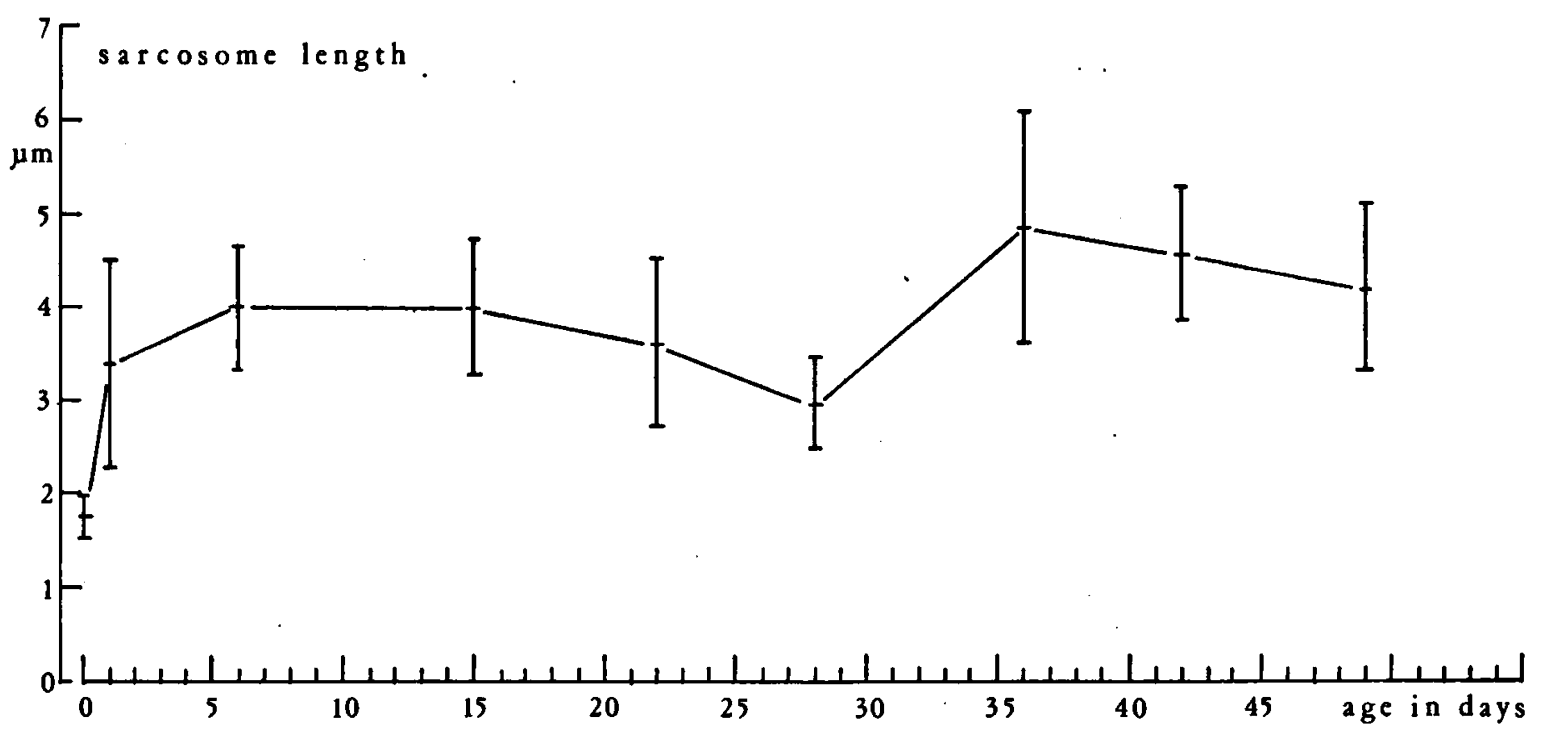

Fig. 9. Sarcosome length in longitudinal sections of flight muscles of Musca domestica at various ages. Bars represent the $95 \%$ confidence limits of the mean.

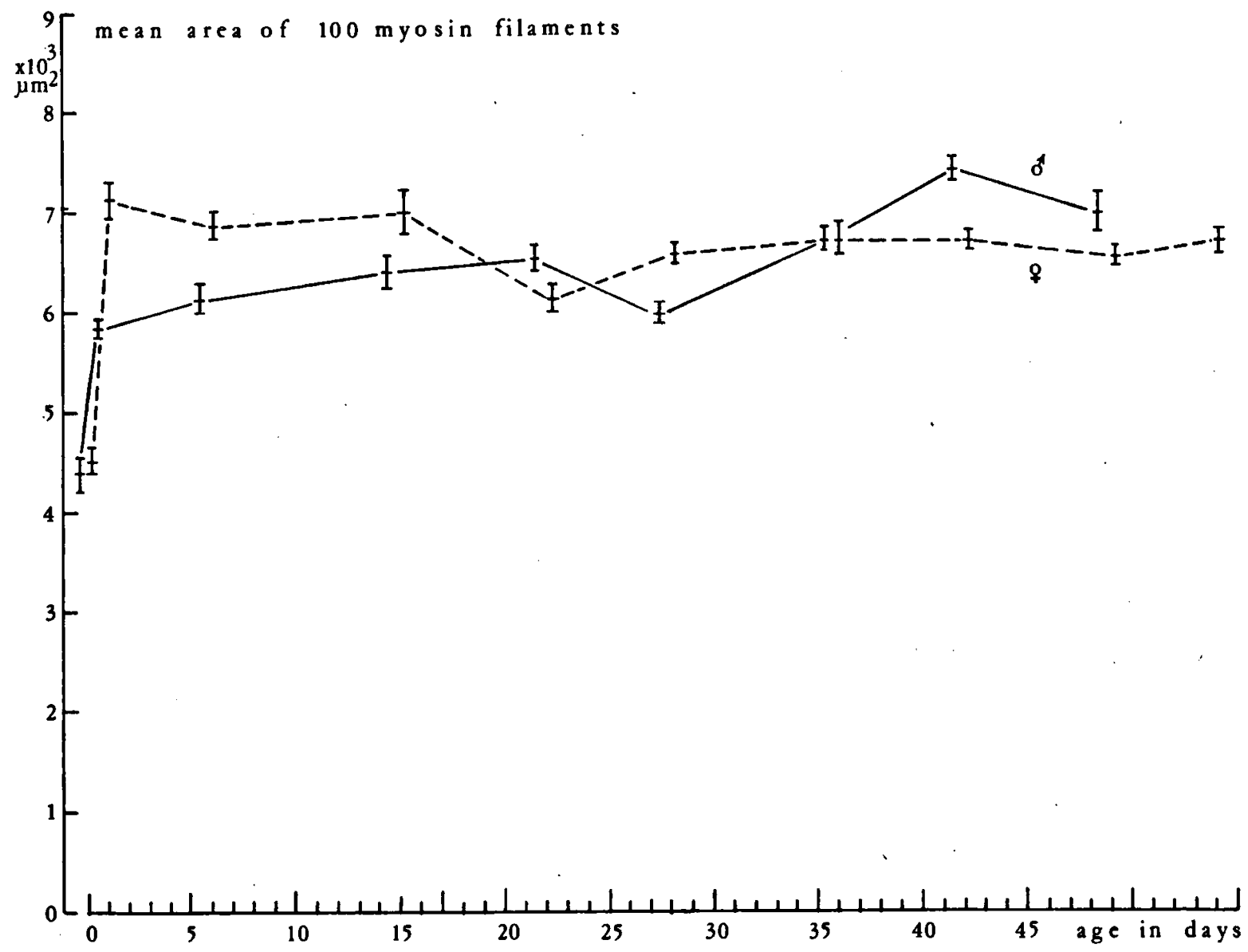

Fig. 10. Mean area of $10 \times 10$ myosin filaments in transverse sections of flight muscles of Musca domestica at various ages, as a measure for the distance between the fibrils. Bars represent the $95 \%$ confidence limits of the mean. 
1969 and Sohal \& Allison, 1971, were primarily due to faulty fixing techniques. It is possible, at an unfavorable osmotic value, that the sarcosomes will break or swell and become lighter in color, as described by Gregory et al., 1968. Weinbach, 1960, remarked that older sarcosomes are less suited for some treatments than younger ones. Electron microscopy can be considered one of these treatments.

The food reserve, at first present in a large quantity, strongly diminishes after the first week and at the end of the second week has completely disappeared from the wing muscles. From Engels', 1968, experiments, carried out under anaerobic conditions, it appears that flies with a food reserve have a greater life expectancy than animals without one. Since the difference in the amount of food reserve available is so slight between males and females, it is difficult to extrapolate the difference to life expectancy. It is, however, possible that the ovaries of older females contain food reserves, as has been observed in other insects. Many small tracheoles were found in flies less than one day old. They are less numerous in older flies. Nevertheless, it appears to have little influence on muscle activity, as young flies are certainly not good flyers.

From our observations on the number of cristae per $\mu \mathrm{m}$, it is not possible to agree with the conclusion of Simon et al., 1969, that there are two types of sarcosomes present. A difference in cristae density is possible in various parts of the sarcosomes. Our value at 28 days of 1 crista per $0.03 \mu \mathrm{m}$ agrees with that found by Smith, 1961, for Calliphora erythrocephala (Meigen, 1826). Sarcosomes or parts of sarcosomes with few cristae are light in color and show up quite clearly. They appear to be quite rare and, thus, their importance in ageing is dubious. Degeneration was not found, even in very old flies (with one exception, perhaps due to sickness).

No visible structural changes during ageing were found. This is in contrast with the observations of Simon et al., 1969, Gregory et al., 1968, and Sohal \& Allison, 1971. Even the quantity of muscle components within the cell gave us little support. The sarcosomes form $30 \%$ of the muscle volume. A comparable percentage was found by Levenbook \& Williams, 1956, in Phormia regina, that is, $32.6 \%$ on a dry weight basis and $40 \%$ on a wet weight basis.

Young flies have a definite advantage as far as the energy supply is concerned. While it is true that the percentage of sarcosomes remains constant during the life span, young flies have small sarcosomes and fibrils, and consequently, a proportionately large "exchange surface". The sarcosomes of males continue growing after 21 days, while those of females then stop growing. This is a disadvantage for the males and perhaps one of the reasons for their shorter life span.

The decline in the size of the sarcosomes, as found by Rockstein, 1966, is perhaps due to a different measuring method. Whenever one determines the size of the sarcosomes from their weight (sedimentation speed), the number of cristae also plays an important role.

Michejda's statement (1964), that the ratio of fibrils to sarcosomes is approximately $1: 1$ for good flyers and $2: 1$ for bad flyers is debatable in the light of the values of 2.2-2.6 found. The ratio of fibrils to sarcosomes, in relation to the energy supply, could possibly play a role in ageing. One can imagine an ideal ratio whereby the energy supply is optimally satisfied. When the optimum has not been reached, animals possessing more sarcosomes than fibrils are favored. In older flies, this ratio is more favorable in females than in males. Perhaps this also contributes to a difference in their life spans. Most of the observations show a change after 28 days. As changes in the distances between myosin filaments also occur between 21-28 days, an artefact might be suspected. In this age group, due to the high mortality rate, random sample mistakes could play a role. A third possibility not to be overlooked is that after 28 days other processes begin. After this time only the strongest specimens survive.

\section{Enzyme activity}

One must be cautious in the interpretation of observations due to the relatively small number of observations and the defective techniques used to localize enzymes electron microscopically. Wohlrab \& Fuchs, 1967, state e.g. that tetrazolium salt TNBT cannot specifically be bound to lipoprotein structures. Sugars, ubichinon, and a few other compounds may cause a nonspecific reaction. Osmium tetroxyde appears to influence the size of the precipitation particles. Attempts to use osmiophilic TC-NBT by Seligman et al., $1967 \&$ 1968, failed due to difficulties with the synthesis of tetrazolium salt. 
Both with tetrazolium salts and ferricyanide we found the same reactions, viz. on the intracristate side of the cristae, as described in literature (Ogawa et al., 1968, Tsou et al., 1968, KerpelFronius \& Hajos, 1968 and Ogawa \& Barnett, 1965). Heterogeneity in the sarcosomes was also observed. We also noticed a heterogeneity in the positive control using rat hearts, although it is doubtful if there are different types of sarcosomes in the wing muscles. A succinate dehydrogenase reaction, however, presents problems. We probably did not succeed in breaking through the barrier for Krebs cycle compounds, whereby the substrate could not sufficiently penetrate. Van den Bergh, 1964, suggests that this barrier is present in order to prevent the Krebs cycle compounds from diffusing out of the sarcosomes. A removal of this barrier is always coupled with structural damages and an increased chance of nonspecific staining. There is no indication whatsoever that any connection exists between the activity of the sarcosomes and their age. Weinbach, 1960, likewise did not see a connection between reaction and age in the sarcosomes of rat livers and brain.

\section{ACKNOWLEDGEMENTS}

The authors wish to thank Prof. Dr. G. Barendrecht, Dr. A. van der Stelt, Mrs. Drs. F. F. J. M. Peeters-Pieters and Drs. W. A. Smit for their valuable comments.

\section{LITERATURE CITED}

Bergh, S. G. van Den, 1964. Pyruvate oxidation and the permeability of Housefly sarcosomes. Biochem. J., 93: 128.

Clark, A. M. \& M. Rockstein, 1964. Ageing in insects. In: M. Rockstein ed., The physiology of insecta, 1: 227 (Academic Press, London).

CURTIS, H. J., 1963. Biological mechanisms underlying the ageing process. Science, 141: $686-694$.

Davies, R. E., 1963. A molecular theory of muscle contraction. Nature, Lond., 199: 1068-1074.

Ebashi, E. \& M. ENDO, 1968. Molecular basis for the regulating action of calcium ions on the contractile system. Progr. Biophys. molec. Biol., 18: 160.

Engels, W., 1968. Anaerobiose Versuche mit Musca domestica. Alters- und Geslechtsunterschiede von Ueberlebensrate und Erholfähigkeit. J. Insect Physiol., 14: 253-260.

Gregory, D. W., R. W. LenNiE \& L. M. BIRT, 1968. An electron-microscopic study of flight muscle development in the Blowfly Lucilia cuprina. J. R. microsc. Soc., 88 (2): $151-175$.
Herold, R. C., 1965. Development und ultrastructural changes of sarcosomes during Honeybee flight muscle development. Devl. Biol., 12: 269-286.

Kerpel-Fronius, S. \& F. HaJos, 1968. The use of ferricyanide for the light and electron microscopic demonstration of succinic dehydrogenase activity. Histochemie, 14: 343-351.

LeVenbook, L. \& C. M. Williams, 1956. Mitochondria in the flight muscles of insects, iii. Mitochondrial cytochrome c in relation to the ageing and wingbeat frequency of flies. J. gen. Physiol., 39: 497-512.

MichejDA, J., 1964. Physiology and structure of flightmuscle sarcosomes in the Silkworm, Hyalophora cecropia. Bull. Soc. Amis Sci. Lett. Poznan (série D.), 4: 61-102.

OGAWA, K. \& R. J. BARNETT, 1965. Electron cytochemical studies of succinic dehydrogenase and dihydronicotinamideadenine dinucleotide diaphorase activities. J. Ultrastruct. Res., 12: 488-508.

Ogawa, K., T. SaIto \& H. Mayahara, 1968. The site of ferricyanide reduction by reductases within mitochondria as studied by electron microscopy. J. Histochem. Cytochem., 16: 49-58.

Rockstein, M., 1966. Biology of ageing insects. In: P. L. KROHN ed., Topics in the biology of ageing: $43-61$. (J. Wiley, London).

Rockstein, M. \& P. L. Bhatnagar, 1965. Age changes in size and number of giant mitochondria in the flight muscle of the Common Housefly. J. Insect Physiol., 11: 481-491.

Seligman, A. M., M. J. Karnovsky, H. L. Wasserkrug \& J. S. HANKER, 1968. Nondroplet ultrastructural demonstration of cytochrome oxidase activity with a polymerizing osmiophilic reagent, diaminobenzedine (DAB). J. Cell Biol., 38 (1): 1.

Seligman, A. M., H. Ueno, Y. Morizono, H. L. WasserKRUG, L. KATZOFF \& J. S. HANKER, 1967. Electron microscopic demonstration of dehydrogenase activity with a new osmiophilic ditetrazolium salt (TC-NBT). J. Histochem. Cytochem., 15 (1): $1-14$.

Simon, J., P. L. Bhatnagar \& N. S. Milburn, 1969. An electron microscope study of changes in mitochondria of flight muscle of ageing Houseflies (Musca domestica). J. Insect Physiol., 15: 135-140.

SMITH, D. S., 1961. The structure of flightmuscle sarcosomes in the Blowfly Calliphora erythrocephala. J. Cell Biol., 19 (4): $115-138$.

Sohal, R. S. \& V. F. Allison, 1971. Age related changes in the fine structure of the flight muscle in the Housefly. Exp. Gerontol., 6: 167-173.

Strehler, B. L., 1962. Time, cells and ageing: 1-270 (Academic Press, London).

Tsou, K. C., E. W. Goodwin, B. Seamond \& D. Lymn, 1968. Intracristal localization of S.D.H.-activity with a new osmium-containing tetra-tetrazolium salt. J. Histochem. Cytochem., 16 (7): 487.

Watanabe, M. I. \& C. M. Williams, 1953. Mitochondria in the flight muscle of insects, ii. Effects of the medium on the size, form and organization of isolated sarcosomes. J. gen. Physiol., 37 (1): 71-90.

WeinBACH, E. C., 1960. Biochemical changes in mitochondria 
associated with age. In: B. L. STREHLER ed., The biology of aging. A symposium held at Gatlinburg, Tennessee, May $1-3,1957$, under the sponsorship of the AIBS and with support of the National Science Foundation. Publs. Am. Inst. biol. Sci., 6: 328-331 (Am. Inst. biol. Sci., Washington).

WohlRAB, F. \& U. Fuchs, 1967. Nichtenzymatische TNBT.

Färbung von Gewebestrukturen. Histochemie, 9: 256-268.

Received: 1 June 1976 